\title{
Estimates of Initial Coefficients for Bi-Univalent Functions
}

\author{
Pawel Zaprawa \\ Department of Mathematics, Lublin University of Technology, Nadbystrzycka 38D, 20-618 Lublin, Poland \\ Correspondence should be addressed to Paweł Zaprawa; p.zaprawa@pollub.pl
}

Received 10 February 2014; Revised 14 April 2014; Accepted 26 April 2014; Published 21 May 2014

Academic Editor: Claudia Timofte

Copyright (C) 2014 Paweł Zaprawa. This is an open access article distributed under the Creative Commons Attribution License, which permits unrestricted use, distribution, and reproduction in any medium, provided the original work is properly cited.

We consider the Fekete-Szegö inequalities for classes which were defined by Murugusundaramoorthy et al. (2013). These inequalities will result in bounds of the third coefficient which are better than these obtained by Murugusundaramoorthy et al. (2013). Moreover, we discuss two other classes of bi-univalent functions. The estimates of initial coefficients in these classes are obtained.

\section{Introduction}

Let $\mathscr{A}$ denote the class of all functions of the form

$$
f(z)=z+\sum_{n=2}^{\infty} a_{n} z^{n}
$$

analytic in the unit disk $\mathbb{D} \equiv\{\zeta \in \mathbb{C}:|\zeta|<1\}$, and let $\mathcal{S}$ denote the class of these functions in $\mathscr{A}$ which are univalent. It is known that if $f \in \mathcal{S}$ then there exists the inverse function $f^{-1}$. Because of the normalization $f(0)=0, f^{-1}$ is defined in some neighbourhood of the origin. In some cases, $f^{-1}$ can be defined in the whole $\mathbb{D}$. Clearly, $f^{-1}$ is also univalent. For this reason, the class $\Sigma$ is defined as follows.

A function $f \in \mathscr{A}$ is called bi-univalent in $\mathbb{D}$ if both $f$ and $f^{-1}$ are univalent in $\mathbb{D}$. The set of all bi-univalent functions is usually denoted by $\Sigma$ (or, following Lewin, by $\sigma$ ).

It is easy to check that a bi-univalent function $f$ given by (1) has the inverse with the Taylor series of the form

$$
f^{-1}(w)=w-a_{2} w^{2}+\left(2 a_{2}^{2}-a_{3}\right) w^{3}+\cdots
$$

The research into $\Sigma$ was started by Lewin ([1], 1967). It focused on problems connected with coefficients. Many papers concerning bi-univalent functions have been published recently. We owe the revival of these topics to Srivastava et al. ([2], 2010). The investigations in this direction have also been carried out, among others, by Ali et al. [3], Frasin and
Aouf [4], and Xu et al. [5]. Hamidi and Jahangiri (e.g., [6]) have revealed the importance of the Faber polynomials in general studies on the coefficients of bi-univalent functions.

In fact, little is known about exact bounds of the initial coefficients of $f \in \Sigma$. For the most general families of functions given by (1) we know that $\left|a_{2}\right|<1.51$ for biunivalent functions (Lewin, [1]), $\left|a_{2}\right| \leq \sqrt{2}$ for bi-starlike functions (Kędzierawski, [7]), and $\left|a_{2}\right| \leq 1$ for bi-convex functions (Brannan and Taha, [8]). Only the last estimate is sharp; equality holds only for $f(z)=z /(1-z)$ and its rotations.

In the papers [1-15], the authors present some estimates for $a_{2}$ and $a_{3}$, while $f$ is taken from various subclasses of $\Sigma$. In 2013 Murugusundaramoorthy et al. (see [16]) obtained some coefficient bounds in two classes: $\delta_{\Sigma}(\alpha, \lambda)$ and $\mathscr{M}_{\Sigma}(\beta, \lambda)$. For a function $f \in \Sigma$ and its inverse function $g$, let $F$ and $G$ be defined as below:

$$
\begin{aligned}
& F(z) \equiv \frac{z f^{\prime}(z)}{(1-\lambda) f(z)+\lambda z f^{\prime}(z)} \\
& G(w) \equiv \frac{w g^{\prime}(w)}{(1-\lambda) g(w)+\lambda w g^{\prime}(w)}
\end{aligned}
$$

where $0 \leq \lambda<1, z \in \mathbb{D}$, and $g=f^{-1}$.

The definitions of $\mathcal{S}_{\Sigma}(\alpha, \lambda)$ and $\mathscr{M}_{\Sigma}(\beta, \lambda)$ are the following. 
Definition 1. A function $f \in \Sigma$ is said to be in the class $\delta_{\Sigma}(\alpha, \lambda)$ if the functions $F$ and $G$, defined by (3), corresponding to $f$ and $g=f^{-1}$, satisfy

$$
\begin{array}{r}
|\arg F(z)|<\frac{\alpha \pi}{2}, \quad|\arg G(w)|<\frac{\alpha \pi}{2}, \\
0<\alpha \leq 1, \quad 0 \leq \lambda<1, \quad z \in \mathbb{D} .
\end{array}
$$

Definition 2. A function $f \in \Sigma$ is said to be in the class $\mathscr{M}_{\Sigma}(\beta, \lambda)$ if the functions $F$ and $G$, defined by (3), corresponding to $f$ and $g=f^{-1}$, satisfy

$$
\begin{gathered}
\mathfrak{R} F(z)>\beta, \quad \mathfrak{R} G(w)>\beta, \\
0 \leq \beta<1, \quad 0 \leq \lambda<1 \quad z \in \mathbb{D} .
\end{gathered}
$$

In particular, for $\lambda=0$, the classes $\mathcal{S}_{\Sigma}(\alpha, \lambda)$ and $\mathscr{M}_{\Sigma}(\beta, \lambda)$ become the class $\mathcal{S}_{\Sigma}^{\alpha}$ of strongly bi-starlike functions of order $\alpha$ and the class $\mathcal{S}_{\Sigma}^{*}(\beta)$ of bi-starlike functions of order $\beta$, respectively. If additionally $\alpha=1$ or $\beta=0$, these two classes reduce to the class $\mathcal{S}_{\Sigma}^{*}$ of bi-starlike functions.

Conditions (4) and (5) in the above definitions can be rewritten as follows:

$$
\begin{aligned}
F(z)=[p(z)]^{\alpha}, & G(w) & =[q(w)]^{\alpha}, \\
F(z)=\beta+(1-\beta) p(z), & G(w) & =\beta+(1-\beta) q(w),
\end{aligned}
$$

respectively, where $p$ and $q$ are functions in $\mathscr{P}$ and have the form

$$
p(z)=1+\sum_{k=1}^{\infty} p_{k} z^{k}, \quad q(w)=1+\sum_{k=1}^{\infty} q_{k} w^{k} .
$$

Throughout the paper, $\mathscr{P}$ stands for the set of all analytic functions $h$ such that $h(0)=1$ and $\Re h(z)>0$ for $z \in \mathbb{D}$.

In [11] the authors proved the following theorems.

Theorem 3. If $f \in \mathcal{S}_{\Sigma}(\alpha, \lambda), 0<\alpha \leq 1$, and $0 \leq \lambda<1$, then

$$
\left|a_{2}\right| \leq \frac{2 \alpha}{(1-\lambda) \sqrt{1+\alpha}}, \quad\left|a_{3}\right| \leq \frac{4 \alpha^{2}}{(1-\lambda)^{2}}+\frac{\alpha}{1-\lambda} .
$$

Theorem 4. If $f \in \mathscr{M}_{\Sigma}(\beta, \lambda), 0 \leq \beta<1$, and $0 \leq \lambda<1$, then

$$
\left|a_{2}\right| \leq \frac{\sqrt{2(1-\beta)}}{1-\lambda}, \quad\left|a_{3}\right| \leq \frac{4(1-\beta)^{2}}{(1-\lambda)^{2}}+\frac{1-\beta}{1-\lambda} .
$$

The above results can be improved. In order to do this, we consider the Fekete-Szegö inequalities for the discussed classes. This type of problems has been considered by many authors. The results concerning this problem are given, for example, in [17-20]. Moreover, it seems to be interesting to discuss two other classes defined in a similar way to $\mathcal{S}_{\Sigma}(\alpha, \lambda)$ and $\mathscr{M}_{\Sigma}(\beta, \lambda)$. The results presented in the paper are not sharp, but, unfortunately, no method which gives sharp results with regard to these problems is known.

In the proofs of the main theorems we need two lemmas.
Lemma 5. If $p(z)=1+\sum_{k=1}^{\infty} p_{k} z^{k} \in \mathscr{P}$ then $\left|p_{k}\right| \leq 2$ for all positive integers $k$.

Lemma 6. Let $k \in \mathbb{R}$ and $z_{1}, z_{2} \in \mathbb{C}$. If $\left|z_{1}\right|<R$ and $\left|z_{2}\right|<R$ then

$$
\left|(k+1) z_{1}+(k-1) z_{2}\right| \leq \begin{cases}2|k| R & \text { for }|k| \geq 1 \\ 2 R & \text { for }|k| \leq 1\end{cases}
$$

The proof of Lemma 6 is easy. It is enough to observe that

$$
\left|(k+1) z_{1}+(k-1) z_{2}\right| \leq(|k+1|+|k-1|) R
$$

and to discuss three cases with respect to $k$.

From Lemma 6 we immediately obtain the following.

Lemma 7. Let $k, l \in \mathbb{R}$ and $z_{1}, z_{2} \in \mathbb{C}$. If $\left|z_{1}\right|<R$ and $\left|z_{2}\right|<R$ then

$$
\left|(k+l) z_{1}+(k-l) z_{2}\right| \leq \begin{cases}2|k| R & \text { for }|k| \geq|l|, \\ 2|l| R & \text { for }|k| \leq|l| .\end{cases}
$$

\section{Results for $\mathcal{S}_{\Sigma}(\alpha, \lambda)$ and $\mathscr{M}_{\Sigma}(\beta, \lambda)$}

Now, we will formulate two theorems concerning the FeketeSzegö inequalities for $\mathcal{S}_{\Sigma}(\alpha, \lambda)$ and $\mathscr{M}_{\Sigma}(\beta, \lambda)$.

Theorem 8. If $f \in \mathcal{S}_{\Sigma}(\alpha, \lambda), 0<\alpha \leq 1,0 \leq \lambda<1$, and $\mu \in \mathbb{R}$, then

$$
\begin{aligned}
& \left|a_{3}-\mu a_{2}{ }^{2}\right| \\
& \leq\left\{\begin{array}{lr}
\frac{4 \alpha^{2}}{(1-\lambda)^{2}(1+\alpha)}|1-\mu| & \text { for } 4 \alpha|1-\mu| \\
\frac{\alpha}{1-\lambda} & \text { for } 4 \alpha|1-\mu| \\
& \leq(1+\alpha)(1-\lambda) .
\end{array}\right.
\end{aligned}
$$

Theorem 9. If $f \in \mathscr{M}_{\Sigma}(\beta, \lambda), 0 \leq \beta<1,0 \leq \lambda<1$, and $\mu \in \mathbb{R}$, then

$$
\left|a_{3}-\mu a_{2}^{2}\right| \leq \begin{cases}\frac{2(1-\beta)}{(1-\lambda)^{2}}|1-\mu| & \text { for } 2|1-\mu| \geq 1-\lambda \\ \frac{1-\beta}{1-\lambda} & \text { for } 2|1-\mu| \leq 1-\lambda .\end{cases}
$$

Proof of Theorem 8. Let $f$ given by (1) be in $\delta_{\Sigma}(\alpha, \lambda)$ and let $0<\alpha \leq 1,0 \leq \lambda<1$, and $\mu \in \mathbb{R}$. From Definition 1 and from (6) we know that

$$
\begin{aligned}
& \frac{z f^{\prime}(z)}{(1-\lambda) f(z)+\lambda z f^{\prime}(z)}=[p(z)]^{\alpha}, \\
& \frac{w g^{\prime}(w)}{(1-\lambda) g(w)+\lambda w g^{\prime}(w)}=[q(w)]^{\alpha},
\end{aligned}
$$

where $p$ and $q$ are functions in $\mathscr{P}$ which have the form (8). 
Comparing the coefficients in each equality in (16), it follows that

$$
\begin{gathered}
(1-\lambda) a_{2}=\alpha p_{1} \\
2(1-\lambda) a_{3}-\left(1-\lambda^{2}\right) a_{2}{ }^{2}=\frac{1}{2} \alpha(\alpha-1) p_{1}{ }^{2}+\alpha p_{2} \\
-(1-\lambda) a_{2}=\alpha q_{1} \\
-2(1-\lambda) a_{3}+\left(\lambda^{2}-4 \lambda+3\right) a_{2}{ }^{2}=\frac{1}{2} \alpha(\alpha-1) q_{1}{ }^{2}+\alpha q_{2}
\end{gathered}
$$

From (17) and (19), there is $p_{1}=-q_{1}$. Summing and subtracting (18) and (20), we have two equalities

$$
\begin{gathered}
2(1-\lambda)^{2} a_{2}^{2}=\frac{1}{2} \alpha(\alpha-1)\left(p_{1}^{2}+q_{1}^{2}\right)+\alpha\left(p_{2}+q_{2}\right), \\
a_{3}=a_{2}^{2}+\frac{\alpha}{4(1-\lambda)}\left(p_{2}-q_{2}\right) .
\end{gathered}
$$

Applying (17) and (19) we dispose of $p_{1}$ and $q_{1}$ in (21). Hence

$$
\begin{aligned}
a_{3}-\mu a_{2}^{2}= & p_{2}\left[h(\alpha)(1-\mu)+\frac{\alpha}{4(1-\lambda)}\right] \\
& +q_{2}\left[h(\alpha)(1-\mu)-\frac{\alpha}{4(1-\lambda)}\right],
\end{aligned}
$$

where $h(\alpha)=\alpha^{2} /(1-\lambda)^{2}(1+\alpha)$ is nonnegative. From Lemmas 5 and 7 we conclude

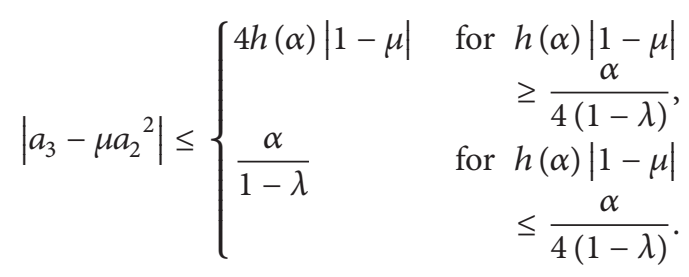

The proof of Theorem 9 is similar to that of Theorem 8 and can be omitted. From Theorems 8 and 9 we get the following corollaries.

Corollary 10. If $f \in \mathcal{S}_{\Sigma}(\alpha, \lambda), 0<\alpha \leq 1$, and $0 \leq \lambda<1$, then

$$
\left|a_{3}\right| \leq \begin{cases}\frac{4 \alpha^{2}}{(1-\lambda)^{2}(1+\alpha)} & \text { for } 4 \alpha \geq(1+\alpha)(1-\lambda) \\ \frac{\alpha}{1-\lambda} & \text { for } 4 \alpha \leq(1+\alpha)(1-\lambda) .\end{cases}
$$

Corollary 11. If $f \in \mathscr{M}_{\Sigma}(\beta, \lambda), 0 \leq \beta<1$, and $0 \leq \lambda<1$, then

$$
\left|a_{3}\right| \leq \frac{2(1-\beta)}{(1-\lambda)^{2}} .
$$

The result in Corollary 10 improves the corresponding result in Theorem 3 . Similarly, for $0 \leq \beta \leq 1 / 2$ the bound in Corollary 11 is better that the one obtained in Theorem 4 .
If $\lambda=0$ we get the bounds for $\mathcal{S}_{\Sigma}^{\alpha}$ and $\mathcal{S}_{\Sigma}^{*}(\beta)$ which are better than these obtained in $[3,11]$. It is worth mentioning that recently Hamidi and Jahangiri ([6]) and Srivastava et al. ([13]) have provided an improvement of the result from Corollaries 10 and 11.

If additionally $\alpha=1$, we obtain that $\left|a_{3}\right| \leq 2$ for the class $\mathcal{S}_{\Sigma}^{*}$ of bi-starlike functions (see $\left.[3,6,13]\right)$.

\section{Results for $\mathscr{K}_{\Sigma}(\alpha, \lambda)$ and $\mathscr{N}_{\Sigma}(\beta, \lambda)$}

To begin with, we can observe that the operators which were used by Murugusundaramoorthy et al. in the definitions of $\mathcal{S}_{\Sigma}(\alpha, \lambda)$ and $\mathscr{M}_{\Sigma}(\beta, \lambda)$ can be written as the weighted harmonic mean of two expressions: $z f^{\prime}(z) / f(z)$ and 1; that is,

$$
\begin{aligned}
& F(z)=\left[\frac{1-\lambda}{z f^{\prime}(z) / f(z)}+\frac{\lambda}{1}\right]^{-1}, \\
& G(w)=\left[\frac{1-\lambda}{w g^{\prime}(w) / g(w)}+\frac{\lambda}{1}\right]^{-1},
\end{aligned}
$$

where $g=f^{-1}$.

Let us define two new classes. In definitions of $F$ and $G$ we consider the weighted harmonic mean of $1+z f^{\prime \prime}(z) / f^{\prime}(z)$ and $z f^{\prime}(z) / f(z)$; namely,

$$
\begin{aligned}
& F(z)=\left[\frac{1-\lambda}{z f^{\prime}(z) / f(z)}+\frac{\lambda}{1+z f^{\prime \prime}(z) / f^{\prime}(z)}\right]^{-1}, \\
& G(w)=\left[\frac{1-\lambda}{w g^{\prime}(w) / g(w)}+\frac{\lambda}{1+w g^{\prime \prime}(w) / g^{\prime}(w)}\right]^{-1},
\end{aligned}
$$

where $0 \leq \lambda \leq 1, z \in \mathbb{D}$, and $g=f^{-1}$. In fact, in the above functions the range of $\lambda$ can be extended to the set $[0, \infty)$.

Now, we can define the classes $\mathscr{K}_{\Sigma}(\alpha, \lambda)$ and $\mathscr{N}_{\Sigma}(\beta, \lambda)$.

Definition 12. A function $f \in \Sigma$ is said to be in the class $\mathscr{K}_{\Sigma}(\alpha, \lambda)$ if the functions $F$ and $G$, defined by (28), corresponding to $f$ and $g=f^{-1}$, satisfy

$$
\begin{array}{r}
|\arg F(z)|<\frac{\alpha \pi}{2}, \quad|\arg G(w)|<\frac{\alpha \pi}{2}, \\
0<\alpha \leq 1, \quad \lambda \geq 0, \quad z \in \mathbb{D} .
\end{array}
$$

Definition 13. A function $f \in \Sigma$ is said to be in the class $\mathcal{N}_{\Sigma}(\beta, \lambda)$ if the functions $F$ and $G$, defined by (28), corresponding to $f$ and $g=f^{-1}$, satisfy

$$
\begin{gathered}
\mathfrak{R} F(z)>\beta, \quad \mathfrak{R} G(w)>\beta, \\
0 \leq \beta<1, \quad \lambda \geq 0, \quad z \in \mathbb{D} .
\end{gathered}
$$

The idea of considering the weighted mean of $1+$ $z f^{\prime \prime}(z) / f^{\prime}(z)$ and $z f^{\prime}(z) / f(z)$ first appeared in the paper by Miller et al. (see [16]). They did their research into the class of so-called $\alpha$-convex functions defined as the arithmetic weighted mean of the expressions mentioned above.

Now we are ready to establish the main theorems of this section. 
Theorem 14. If $f \in \mathscr{K}_{\Sigma}(\alpha, \lambda), 0<\alpha \leq 1, \lambda \geq 0$, and $\mu \in \mathbb{R}$, then

(1)

$$
\left|a_{2}\right| \leq \frac{2 \alpha}{\sqrt{\alpha(1-\lambda)^{2}+(1+\lambda)^{2}}}
$$

$$
\begin{aligned}
& \left|a_{3}-\mu a_{2}{ }^{2}\right| \\
& \leq\left\{\begin{array}{cc}
\frac{4 \alpha^{2}}{\alpha(1-\lambda)^{2}+(1+\lambda)^{2}}|1-\mu| & \text { for } 4 \alpha(1+2 \lambda)|1-\mu| \\
& \geq \alpha(1-\lambda)^{2} \\
\frac{\alpha}{1+2 \lambda} & +(1+\lambda)^{2} \\
& \text { for } 4 \alpha(1+2 \lambda)|1-\mu| \\
& \leq \alpha(1-\lambda)^{2} \\
& +(1+\lambda)^{2} .
\end{array}\right.
\end{aligned}
$$

Theorem 15. If $f \in \mathcal{N}_{\Sigma}(\beta, \lambda), 0 \leq \beta<1, \lambda \geq 0$, and $\mu \in \mathbb{R}$, then

(1)

$$
\left|a_{2}\right| \leq \begin{cases}\frac{2(1-\beta)}{1+\lambda} & \text { for } \beta \geq \frac{(1-\lambda)^{2}}{2\left(1+\lambda^{2}\right)}, \\ \sqrt{\frac{2(1-\beta)}{1+\lambda^{2}}} & \text { for } \beta \leq \frac{(1-\lambda)^{2}}{2\left(1+\lambda^{2}\right)}\end{cases}
$$

(2)

$$
\left|a_{3}-\mu a_{2}^{2}\right| \leq\left\{\begin{array}{lc}
\frac{2(1-\beta)}{1+\lambda^{2}}|1-\mu| & \text { for } 2(1+2 \lambda)|1-\mu| \\
\frac{1-\beta}{1+2 \lambda} & \text { for } 2(1+2 \lambda)|1-\mu| \\
& \leq 1+\lambda^{2} .
\end{array}\right.
$$

Proof of Theorem 14. Assume that $0<\alpha \leq 1, \lambda \geq 0$, and $\mu \epsilon$ $\mathbb{R}$. From Definition 12 it follows that if $f \in \mathscr{K}_{\Sigma}(\alpha, \lambda)$ then

$$
\begin{gathered}
\frac{z f^{\prime}(z)\left[f^{\prime}(z)+z f^{\prime \prime}(z)\right]}{\lambda z\left(f^{\prime}(z)\right)^{2}+(1-\lambda) f(z)\left[f^{\prime}(z)+z f^{\prime \prime}(z)\right]}=[p(z)]^{\alpha}, \\
\frac{w g^{\prime}(z)\left[g^{\prime}(w)+w g^{\prime \prime}(z)\right]}{\lambda w\left(g^{\prime}(z)\right)^{2}+(1-\lambda) g(w)\left[g^{\prime}(w)+w g^{\prime \prime}(w)\right]}=[q(w)]^{\alpha},
\end{gathered}
$$

where $p$ and $q$ are functions in $\mathscr{P}$ and have the form (8).

Hence, comparing the coefficients in each equality in (35), we can write

$$
(1+\lambda) a_{2}=\alpha p_{1}
$$

$$
\begin{gathered}
2(1+2 \lambda) a_{3}+4 a_{2}^{2}-(5-\lambda) a_{2} \alpha p_{1} \\
=\frac{1}{2} \alpha(\alpha-1) p_{1}{ }^{2}+\alpha p_{2}, \\
-(1+\lambda) a_{2}=\alpha q_{1}, \\
-2(1+2 \lambda) a_{3}+8(1+\lambda) a_{2}{ }^{2}+(5-\lambda) a_{2} \alpha q_{1} \\
=\frac{1}{2} \alpha(\alpha-1) q_{1}{ }^{2}+\alpha q_{2} .
\end{gathered}
$$

From (36) and (38), it yields that $p_{1}=-q_{1}$. Putting (36) into (37) and (38) into (39), we obtain

$$
\begin{aligned}
& 2(1+2 \lambda) a_{3}+\left(\lambda^{2}-4 \lambda-1\right) a_{2}{ }^{2}=\frac{1}{2} \alpha(\alpha-1) p_{1}{ }^{2}+\alpha p_{2} \\
& -2(1+2 \lambda) a_{3}+\left(\lambda^{2}+4 \lambda+3\right) a_{2}^{2}=\frac{1}{2} \alpha(\alpha-1) q_{1}{ }^{2}+\alpha q_{2} .
\end{aligned}
$$

Now, summing and subtracting (40) we have two equalities

$$
\begin{aligned}
2\left(1+\lambda^{2}\right) a_{2}^{2} & =\frac{1}{2} \alpha(\alpha-1)\left(p_{1}^{2}+\mathrm{q}_{1}^{2}\right)+\alpha\left(p_{2}+q_{2}\right) \\
a_{3} & =a_{2}^{2}+\frac{\alpha}{4(1+2 \lambda)}\left(p_{2}-q_{2}\right) .
\end{aligned}
$$

Substituting in (41) $p_{1}$ and $q_{1}$ taken from (36) and (38), we get

$$
a_{2}^{2}=\frac{\alpha^{2}}{\alpha(1-\lambda)^{2}+(1+\lambda)^{2}}\left(p_{2}+q_{2}\right) .
$$

By Lemma 5, the first part of our assertion follows.

From (41) and (42),

$$
\begin{aligned}
& a_{3}-\mu a_{2}{ }^{2} \\
& =p_{2}\left[\frac{\alpha^{2}}{\alpha(1-\lambda)^{2}+(1+\lambda)^{2}}(1-\mu)+\frac{\alpha}{4(1+2 \lambda)}\right] \\
& \quad+q_{2}\left[\frac{\alpha^{2}}{\alpha(1-\lambda)^{2}+(1+\lambda)^{2}}(1-\mu)-\frac{\alpha}{4(1+2 \lambda)}\right] .
\end{aligned}
$$

Applying Lemmas 5 and 7 completes the second part of the assertion.

Theorem 14 gives the following corollaries.

Corollary 16. If $f \in \mathscr{K}_{\Sigma}(\alpha, \lambda), 0<\alpha \leq 1$, and $\lambda \geq 0$, then

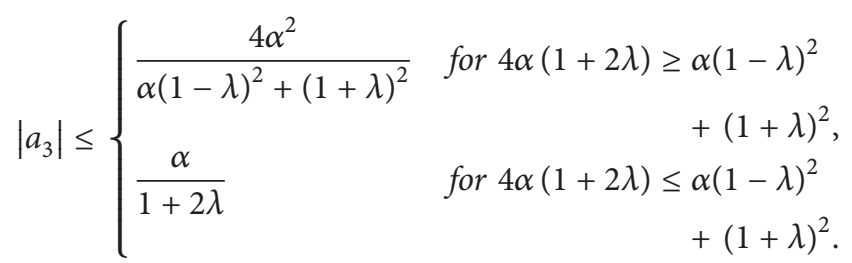


For $\lambda=0$ we obtain the bounds for $\delta_{\Sigma}^{\alpha}$. For $\lambda=1$, the class $\mathscr{K}_{\Sigma}(\alpha, \lambda)$ reduces to the class $\mathscr{K}_{\Sigma}^{\alpha}$ of strongly bi-convex functions of order $\alpha$. Hence we have the following.

Corollary 17. If $f \in \mathscr{K}_{\Sigma}^{\alpha}$ and $0<\alpha \leq 1$, then

(1)

$$
\left|a_{2}\right| \leq \alpha
$$

(2)

$$
\left|a_{3}\right| \leq \begin{cases}\frac{\alpha}{3} & \text { for } \alpha \in\left(0, \frac{1}{3}\right] \\ \alpha^{2} & \text { for } \alpha \in\left[\frac{1}{3}, 1\right]\end{cases}
$$

This result improves the result given in [3].

Proof of Theorem 15. Let $f \in \mathcal{N}_{\Sigma}(\alpha, \lambda)$ with $0 \leq \beta<1$ and $\lambda \geq 0$. From Definition 13 we obtain

$$
\begin{aligned}
& \frac{z f^{\prime}(z)\left[f^{\prime}(z)+z f^{\prime \prime}(z)\right]}{\lambda z\left(f^{\prime}(z)\right)^{2}+(1-\lambda) f(z)\left[f^{\prime}(z)+z f^{\prime \prime}(z)\right]} \\
& =\beta+(1-\beta) p(z), \\
& \frac{w g^{\prime}(z)\left[g^{\prime}(w)+w g^{\prime \prime}(z)\right]}{\lambda w\left(g^{\prime}(z)\right)^{2}+(1-\lambda) g(w)\left[g^{\prime}(w)+w g^{\prime \prime}(w)\right]} \\
& =\beta+(1-\beta) q(w),
\end{aligned}
$$

where $p, q \in \mathscr{P}$.

Hence,

$$
\begin{gathered}
(1+\lambda) a_{2}=(1-\beta) p_{1} \\
2(1+2 \lambda) a_{3}+4 a_{2}^{2}-(5-\lambda)(1-\beta) a_{2} p_{1}=(1-\beta) p_{2} \\
-(1+\lambda) a_{2}=(1-\beta) q_{1} \\
2(1+2 \lambda)\left(2 a_{2}^{2}-a_{3}\right)+4 a_{2}^{2} \\
+(5-\lambda)(1-\beta) a_{2} q_{1}=(1-\beta) q_{2}
\end{gathered}
$$

Applying the same method as in the proof of Theorem 15 we get

$$
\begin{gathered}
a_{2}^{2}=\frac{1-\beta}{2\left(1+\lambda^{2}\right)}\left(p_{2}+q_{2}\right), \\
a_{3}=a_{2}^{2}+\frac{1-\beta}{4(1+2 \lambda)}\left(p_{2}-q_{2}\right) .
\end{gathered}
$$

Observe that the estimate for $\left|a_{2}\right|$ obtained from (53), that is, $\left|a_{2}\right| \leq \sqrt{2(1-\beta) /\left(1+\lambda^{2}\right)}$, is not always better than the estimate which follows directly from (49), that is, $\left|a_{2}\right| \leq 2$ (1$\beta) /(1+\lambda)$. The comparison of these two bounds completes the first part of the proof.
From (53) and (54),

$$
\begin{aligned}
a_{3}-\mu a_{2}{ }^{2}= & p_{2}\left[\frac{(1-\beta)(1-\mu)}{2\left(1+\lambda^{2}\right)}+\frac{1-\beta}{4(1+2 \lambda)}\right] \\
& +q_{2}\left[\frac{(1-\beta)(1-\mu)}{2\left(1+\lambda^{2}\right)}-\frac{1-\beta}{4(1+2 \lambda)}\right],
\end{aligned}
$$

which results in the second part of the assertion.

From Theorem 15 we get the following corollaries.

Corollary 18. If $f \in \mathcal{N}_{\Sigma}(\beta, \lambda), 0 \leq \beta<1$, and $\lambda \geq 0$, then $\left|a_{3}\right| \leq 2(1-\beta) /\left(1+\lambda^{2}\right)$.

For $\lambda=0$ we obtain the bounds for $\mathcal{S}_{\Sigma}^{*}(\beta)$. For $\lambda=1$, the set $\mathscr{N}_{\Sigma}(\beta, \lambda)$ is the class $\mathscr{K}_{\Sigma}(\beta)$ of bi-convex functions of order $\beta$. Hence we have the following.

Corollary 19. If $f \in \mathscr{K}_{\Sigma}(\beta)$ and $0 \leq \beta<1$, then

(1)

$$
\left|a_{2}\right| \leq 1-\beta
$$

(2)

$$
\left|a_{3}\right| \leq 1-\beta
$$

This bound is better than the one proved in [3].

\section{Conflict of Interests}

The author declares that there is no conflict of interests regarding the publication of this paper.

\section{References}

[1] M. Lewin, "On a coefficient problem for bi-univalent functions," Proceedings of the American Mathematical Society, vol. 18, pp. 63-68, 1967.

[2] H. M. Srivastava, A. K. Mishra, and P. Gochhayat, "Certain subclasses of analytic and bi-univalent functions," Applied Mathematics Letters, vol. 23, no. 10, pp. 1188-1192, 2010.

[3] R. M. Ali, S. K. Lee, V. Ravichandran, and S. Supramaniam, "Coefficient estimates for bi-univalent Ma-Minda starlike and convex functions," Applied Mathematics Letters, vol. 25, no. 3, pp. 344-351, 2012.

[4] B. A. Frasin and M. K. Aouf, "New subclasses of bi-univalent functions," Applied Mathematics Letters, vol. 24, no. 9, pp. 15691573, 2011.

[5] Q.-H. Xu, H.-G. Xiao, and H. M. Srivastava, "A certain general subclass of analytic and bi-univalent functions and associated coefficient estimate problems," Applied Mathematics and Computation, vol. 218, pp. 11461-11465, 2012.

[6] S. G. Hamidi and J. M. Jahangiri, "Faber polynomial coefficient estimates for analytic bi-close-to-convex functions," Comptes Rendus de l'Académie des Sciences: Series I, vol. 352, pp. 17-20, 2014.

[7] A. W. Kędzierawski, "Some remarks on bi-univalent functions," nnales Universitatis Mariae Curie-Skłodowska. Sectio A, vol. 39, pp. 77-81, 1985. 
[8] D. A. Brannan and T. S. Taha, "On some classes of biunivalent functions," in Mathematical Analysis and Its Applications, Kuwait, February 1985, S. M. Mazhar, A. Hamoui, and N. S. Faour, Eds., vol. 3 of KFAS Proceedings Series, pp. 53-60, Pergamon Press, Elsevier Science, Oxford, UK, 1988.

[9] M. Çağlar, H. Orhan, and N. Yağmur, "Coefficient bounds for new subclasses of bi-univalent functions," Filomat, vol. 27, pp. 1165-1171, 2013.

[10] S. S. Kumar, V. Kumar, and V. Ravichandran, "Estimates for the initial coefficients of bi-univalent functions," Tamsui Oxford Journal of Information and Mathematical Science. In press.

[11] G. Murugusundaramoorthy, N. Magesh, and V. Prameela, "Coefficient bounds for certain subclasses of bi-univalent function," Abstract and Applied Analysis, vol. 2013, Article ID 573017, 3 pages, 2013.

[12] H. M. Srivastava, "Some inequalities and other results associated with certain subclasses of univalent and bi-univalent analytic functions," in Nonlinear Analysis: Stability, Approximation, and Inequalities, P. M. Pardalos, P. G. Georgiev, and H. M. Srivastava, Eds., vol. 68 of Springer Series on Optimization and Its Applications, pp. 607-630, Springer, Berlin, Germany, 2012.

[13] H. M. Srivastava, S. Bulut, M. Çağlar, and N. Yağmur, "Coefficient estimates for a general subclass of analytic and biunivalent functions," Filomat, vol. 27, pp. 831-842, 2013.

[14] D. Styer and D. J. Wright, "Results on bi-univalent functions," Proceedings of the American Mathematical Society, vol. 82, pp. 243-248, 1981.

[15] Q.-H. Xu, Y.-C. Gui, and H. M. Srivastava, "Coefficient estimates for a certain subclass of analytic and bi-univalent functions," Applied Mathematics Letters, vol. 25, no. 6, pp. 990-994, 2012.

[16] S. S. Miller, P. Mocanu, and M. O. Reade, "All $\alpha$-convex functions are univalent and starlike," Proceedings of the American Mathematical Society, vol. 37, pp. 553-554, 1973.

[17] R. R. London, "Fekete-Szegö inequalities for close-to-convex functions," Proceedings of the American Mathematical Society, vol. 117, no. 4, pp. 947-950, 1993.

[18] H. M. Srivastava, A. K. Mishra, and M. K. Das, "The FeketeSzegö problem for a subclass of close-to-convex functions," Complex Variables, Theory and Application, vol. 44, pp. 145-163, 2001.

[19] J. H. Choi, Y. C. Kim, and T. Sugawa, "A general approach to the Fekete-Szegö problem," Journal of the Mathematical Society of Japan, vol. 59, no. 3, pp. 707-727, 2007.

[20] S. Kanas, "An unified approach to the Fekete-Szegö problem," Applied Mathematics and Computation, vol. 218, no. 17, pp. 8453-8461, 2012. 


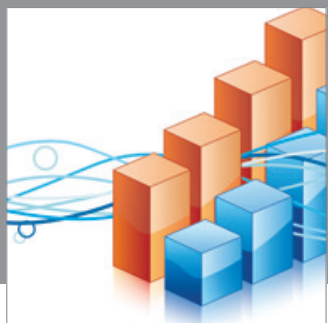

Advances in

Operations Research

mansans

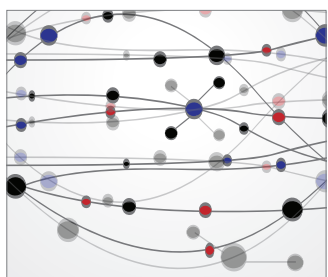

The Scientific World Journal
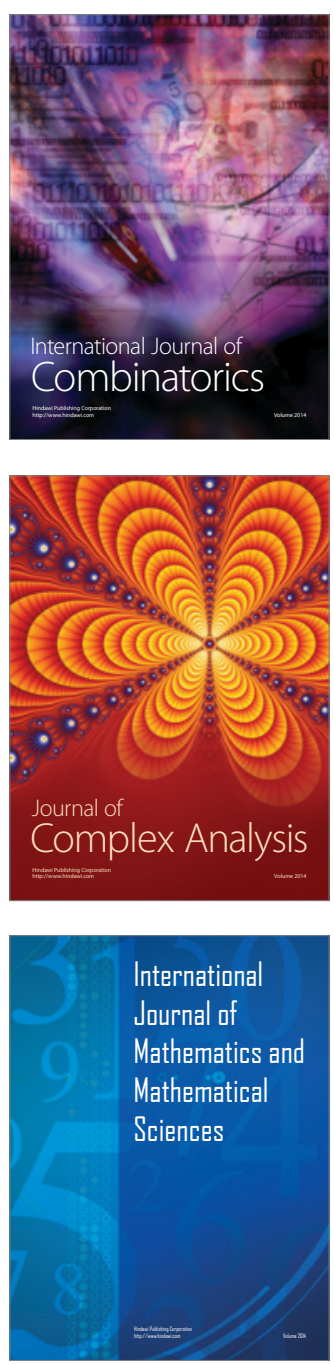
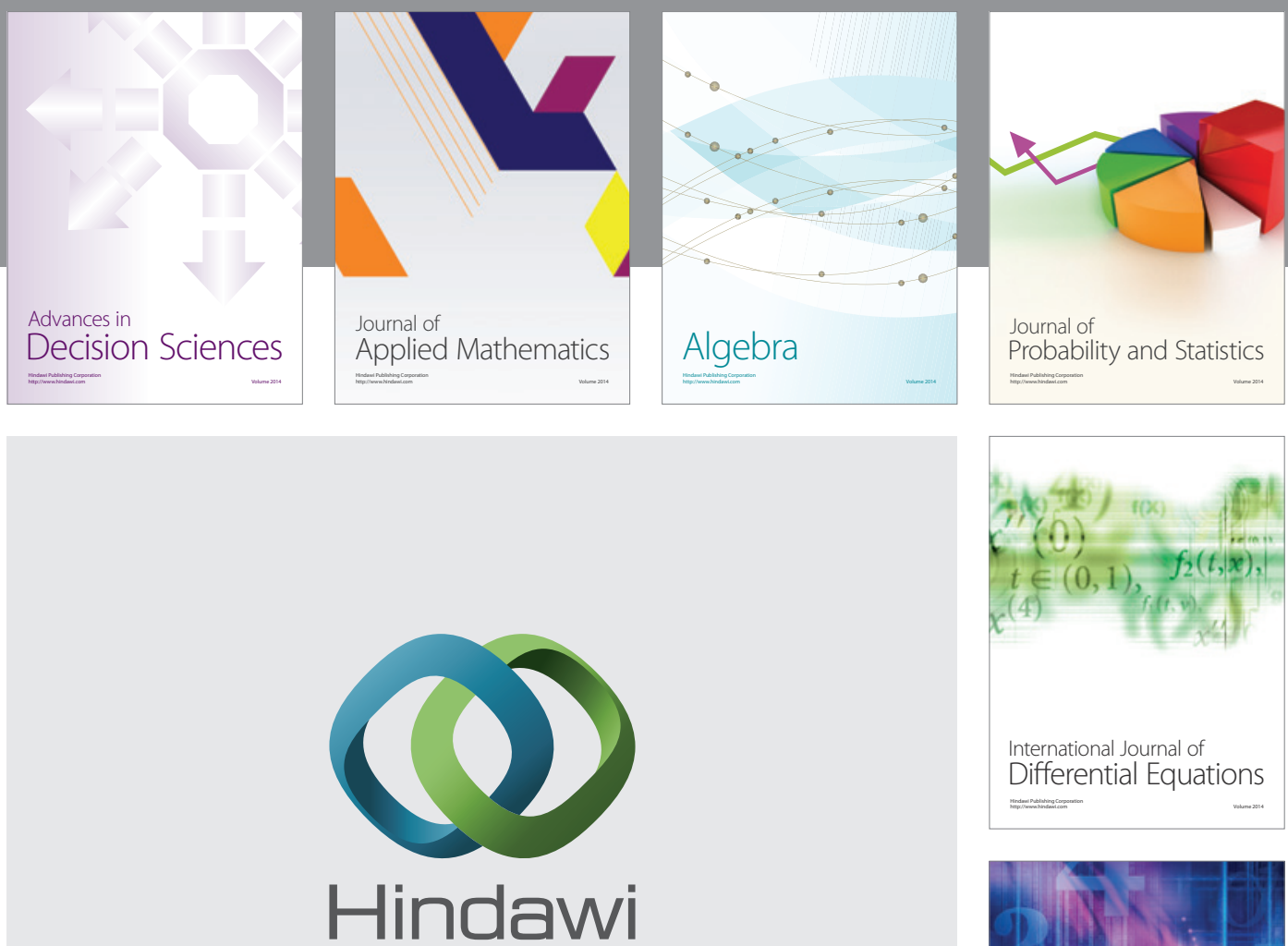

Submit your manuscripts at http://www.hindawi.com
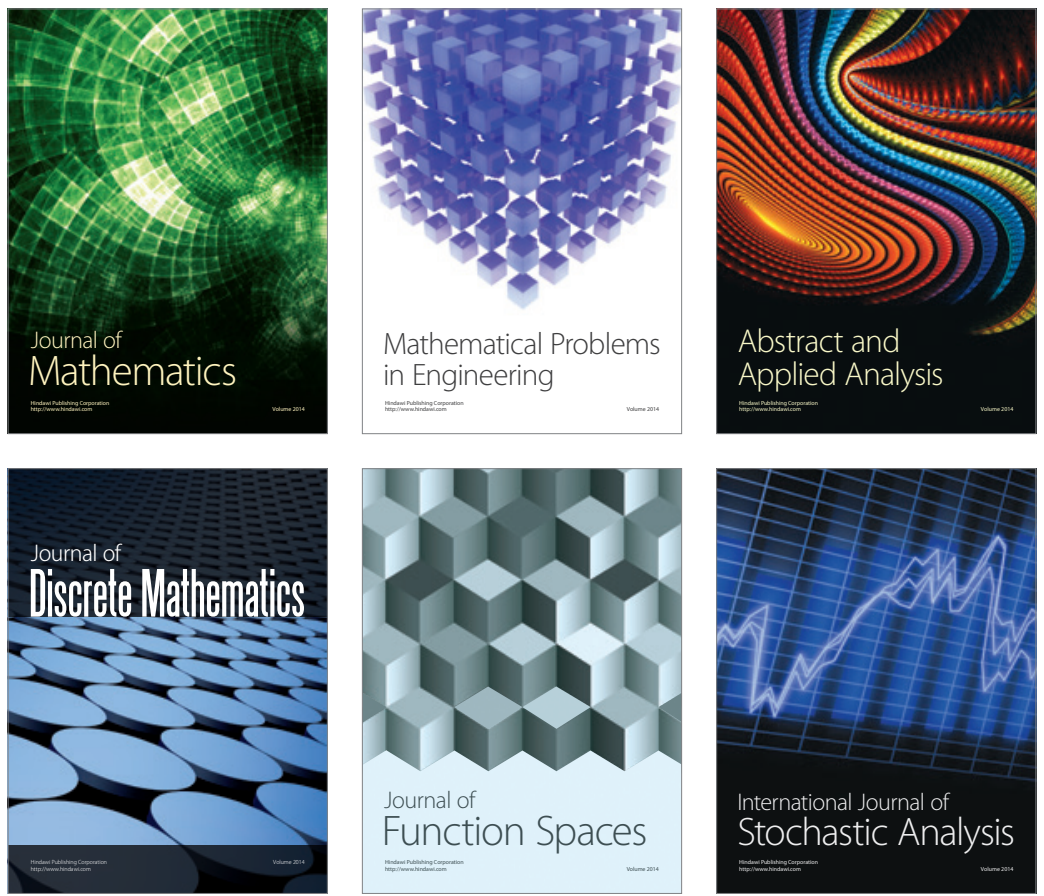

Journal of

Function Spaces

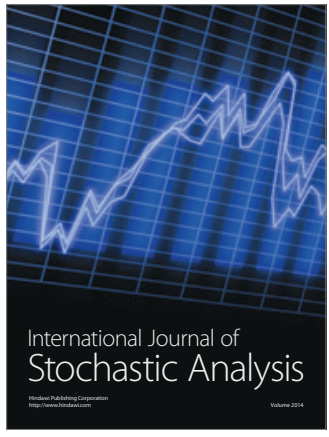

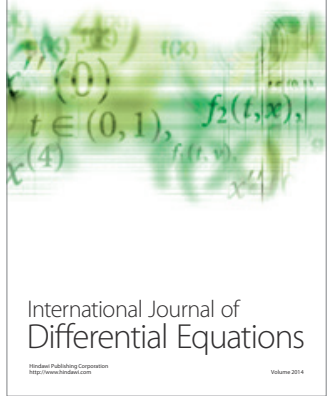
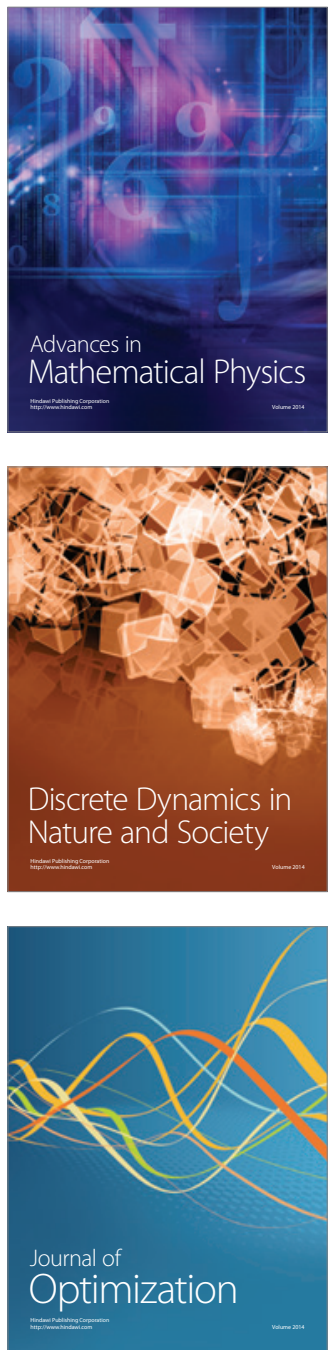